\title{
Bestimmt das Angebot die Förderquote? - Effekte der räumlichen Nähe von Förderschulen auf den Anteil von Schülerinnen und Schülern mit Förderbedarf an Grundschulen
}

\author{
Janka Goldan $(\mathbb{D}) \cdot$ Michael Grosche $(\mathbb{D})$
}

Eingegangen: 23. Juli 2020 / Überarbeitet: 2. November 2020 / Angenommen: 10. März 2021 / Online publiziert: 15. März 2021

(C) Der/die Autor(en) 2021

Zusammenfassung In dem vorliegenden Beitrag wird erstmals umfassend empirisch untersucht, ob vorhandene Angebotsstrukturen in Form von Förderschulen einen systematischen Einfluss auf die Zahl der als sonderpädagogisch förderbedürftig diagnostizierten Schülerinnen und Schüler an naheliegenden Grundschulen haben. Die Hypothese wird systemtheoretisch hergeleitet und anhand von statistischen und wissenschaftlichen Befunden verdeutlicht. Datenbasis bildet die amtliche Schulstatistik Nordrhein-Westfalens für die Grundschulen der Jahre 2010/11 bis 2017/18. Zur Überprüfung der Annahme, dass der Anteil der Schülerinnen und Schüler mit einem formal diagnostizierten Förderbedarf an Grundschulen höher ist, je geringer die räumliche Distanz zur nächstgelegenen Förderschule ausfällt, wird ein Tobit-Regressionsmodell unter Berücksichtigung der Mehrebenenstruktur der Daten berechnet. Die Ergebnisse zeigen für verschiedene Förderschwerpunkte - unter Kontrolle konfundierender Variablen - signifikante Effekte und bestätigen die Annahme. Mögliche Mechanismen des Effekts und Implikationen für die Praxis amtlicher Feststellungsverfahren werden vor dem Hintergrund der schulischen Inklusion beleuchtet.

Schlüsselwörter Inklusion · Systemtheorie · Sonderpädagogischer Förderbedarf · Schulstatistik $\cdot$ Förderschulen

Dr. J. Goldan $(\triangle)$

Fakultät für Erziehungswissenschaft, AG 3 ,Schultheorie mit dem Schwerpunkt Grund- und Förderschulen“, Universität Bielefeld, Postfach 100131, 33615 Bielefeld, Deutschland E-Mail: janka.goldan@uni-bielefeld.de

Prof. Dr. M. Grosche

Professur für „Rehabilitationswissenschaften mit dem Förderschwerpunkt Lernen“ am Institut für Bildungsforschung (IfB), Bergische Universität Wuppertal, Gaußstr. 20, 42097 Wuppertal, Deutschland E-Mail: michael.grosche@uni-wuppertal.de 


\title{
Does the supply determine the quota?-Effects of the geographical proximity of special schools on the number of students with special needs in primary schools
}

\begin{abstract}
The present paper is the first comprehensive empirical study to examine whether an available supply of special schools has a systematic influence on the number of students diagnosed with special educational needs (SEN) in nearby primary schools. The hypothesis is derived from systems theory and substantiated by statistical numbers and scientific findings. Data are based on the official school statistics of the federal state of North Rhine-Westphalia for the years 2010/11 to $2017 / 18$. To test the assumption that the proportion of students with SEN in primary schools is to be higher, the closer the distance to the nearest special school, a Tobit regression model is calculated considering the multi-level structure of the data. The results show significant negative effects for different categories of SEN-controlled by confounding variables - and thus confirm the hypothesis. Potential mechanisms of the effect and implications for the practice of formal SEN assessments are discussed with regard to inclusive education reforms.
\end{abstract}

Keywords Inclusive education - School Statistics - Special Educational Needs · Special Schools $\cdot$ Systems Theory

\section{Einleitung}

Schulstatistische Analysen zu Schülerinnen und Schülern mit einem amtlich diagnostizierten sonderpädagogischen Förderbedarf (SPF) zeigen seit Jahrzehnten erhebliche regionale Unterschiede in der Prävalenz von Förderbedarfen (Autorengruppe Bildungsberichterstattung 2014, 2018, 2020; Klemm 2015, 2018). Die Prävalenzen variieren über die Schuljahre, Förderorte, Förderschwerpunkte, Bundesländer und auch Kommunen hinweg so erheblich, dass Katzenbach (2015, S. 38) von einer „,systematischen Zufälligkeit der Vergabe des Labels ,Sonderpädagogischer Förderbedarf"“ spricht. Zwar sind die Unterschiede zum Teil auf eine regional bedingte Heterogenität der Schülerschaft und unterschiedliche Regelungen in den Feststellungsverfahren der Förderbedarfe zurückzuführen (Autorengruppe Bildungsberichterstattung 2020; Klemm 2015). Dies kann allerdings nicht die gesamte regionale Varianz in der Prävalenz von Förderbedarfen erklären (Katzenbach 2015; Orthmann Bless 2007).

Da die Kriterien zur Diagnose sonderpädagogischer Förderbedarfe ,nur vage bestimmt“ (Orthmann Bless 2007, S. 97) sind (Katzenbach 2015), entsteht ein Ermessensspielraum, der den Einfluss weiterer Faktoren auf die Feststellung von Förderbedarfen begünstigt. In der Literatur werden verschiedene solcher Einflussfaktoren diskutiert, beispielsweise Ressourcen-Etikettierungs-Interessen (Bleidick 1985; Kottmann 2007), Referenzgruppen-Effekte (Koßmann 2019), Migrationshintergrund (Kornmann 2013; gegenteiliger Befund siehe: Kölm et al. 2019), Arbeits- und Sozialverhalten der Schülerinnen und Schüler (Smeets und Roeleveld 2016), Faktoren auf Ebene der Lehrkraft, z. B. Belastbarkeit und Toleranzgrenze (Orthmann Bless 
2007), sowie der Einfluss der Schulkultur und unterschiedliche Bewilligungsprozesse und -verfahren der Bundesländer bzw. zuständigen Schulämter (Neumann und Lütje-Klose 2020). Darüber hinaus können systemgeleitete Interessen bei der Feststellung von Förderbedarfen eine Rolle spielen (Orthmann Bless 2007), z. B. wenn aufgrund der Diagnose besondere Unterstützungsstrukturen in Schulnähe in Anspruch genommen werden können. Theoretisch lässt sich diese Annahme aus der Systemtheorie nach Luhmann (2002) herleiten.

Das Ziel der vorliegenden Studie besteht darin, diese systemtheoretische Annahme erstmalig unter Kontrolle möglicher konfundierender Variablen regressionsanalytisch zu überprüfen. Anhand von Daten der amtlichen Schulstatistik für das Bundesland Nordrhein-Westfalen (Schuljahre 2010/11 bis 2017/18) werden wir untersuchen, ob der Anteil der Schülerinnen und Schüler mit amtlich festgestelltem sonderpädagogischem Förderbedarf an Grundschulen umso höher ausfällt, je geringer die Distanz zur nächsten Förderschule ist. Hierbei kontrollieren wir mithilfe der RWI GEO-GRID Daten (Breidenbach und Eilers 2018) auch für sozialräumliche Einflussfaktoren, z. B. Bevölkerungsdichte und sozio-ökonomische Variablen.

\section{Theoretischer Hintergrund und Forschungsstand}

\subsection{Die Entstehung von Förderbedarfen aus schulsystemischer Sicht}

Zur Erklärung der Entstehung von Förderbedarfen existieren verschiedene Theorien, wie beispielsweise das bio-psycho-soziale Modell, welches die Entstehung von Förderbedarfen v.a. durch ein ungünstiges Zusammenspiel von individuellen und sozialen Faktoren erklärt (z.B. Kretschmann 2007). Daneben existieren Theorien, welche systemgeleitete Interessen stärker betonen und sich der Systemtheorie bedienen (z. B. Werning und Lütje-Klose 2016). Aus schulsystemischer Sicht liegt der Fokus auf der Frage, inwieweit die Strukturen und Normen der (Sub-)Systeme an der Entstehung und Erzeugung von Förderbedarfen mit beteiligt sind (Orthmann Bless 2007).

Aus einer systemtheoretischen Perspektive werden Institutionen als soziale Systeme verstanden (Orthmann Bless 2007; Werning und Lütje-Klose 2016), d.h. im Kontext von Bildung und Schule ist das Schulsystem den sozialen Systemen zuzurechnen (Luhmann 2002). Noch spezifischer lassen sich das Regelschulsystem und das Förderschulsystem als zwei Subsysteme des Schulsystems verstehen (Lambrecht 2020), die - wie alle Systeme - nach Selbsterhalt und zu diesem Zweck u. a. nach Komplexitätsreduktion streben. Beispielsweise erhöht sich die Komplexität des Regelschulsystems, wenn einzelne Schülerinnen und Schüler längerfristig hinter den Anforderungen des Systems Regelschule zurückbleiben, d.h. es wird für das System zu einer wachsenden Herausforderung, die Schülerinnen und Schüler entsprechend geltender Bildungsstandards zu qualifizieren. In der Folge ist das System bestrebt, Komplexität zu reduzieren und wieder einen Gleichgewichtszustand herzustellen, z. B. indem diesen Schülerinnen und Schülern ein sonderpädagogischer Förderbedarf zugewiesen wird, für die bis zur Einführung der schulischen Inklusion ausschließlich das Förderschulsystem zuständig wurde. „Dabei geht man davon aus, 
dass die Konstituierung der Personengruppe [...] [der Schülerinnen und Schüler mit SPF; Anm. d. Autoren] und ihre Zuweisung zu einem Subsystem, also die segmentierte Bearbeitung von Lebensbedürfnissen und Lebensrisiken, das System entlastet und stabilisiert." (Orthmann Bless 2007, S. 94) Dementsprechend kann die Entstehung von Förderbedarfen aus systemtheoretischer Perspektive als eine Folge des selektiven Schulsystems betrachtet werden, dessen Strukturen und Normen an der Entstehung und Erzeugung von Förderbedarfen mit beteiligt sind (Orthmann Bless 2007; Werning und Lütje-Klose 2016).

Zwar stellte die gesetzliche Einführung der schulischen Inklusion im Jahr 2009 in Deutschland die Existenz von Förderschulen kurzzeitig in Frage. Dennoch zeigt sich im Lichte der aktuellen Umsetzung von Inklusion, dass beide Systeme (Regelund Förderschulen) parallel fortbestehen. Demnach sind, wie Lambrecht (2020) darlegt, Förderschulen im Sinne des Selbsterhalts weiter bestrebt, ihre Schülerschaft zu „suchen“. Regelschulen wiederum müssen diese „besonderen“ Schülerinnen und Schüler weiterhin diagnostizieren, um deren mangelnde Passung zum allgemeinen Schulsystem zu signalisieren. Daher ist davon auszugehen, dass die soeben beschriebenen Effekte auch an inklusiven Schulen wirksam werden. Für den Primarbereich zeigen schulstatistische Analysen, dass Schülerinnen und Schüler mit einem amtlich festgestellten Förderbedarf überwiegend an den Grundschulen verbleiben und seltener auf Förderschulen überwiesen werden. Gleichzeitig sind die amtlichen Diagnosen nach wie vor nötig, um Schülerinnen und Schüler an eine Förderschule zu überweisen. Die relativ stabilen Förderschulbesuchsquoten indizieren, dass die Diagnosen zu diesem Zweck auch weiter genutzt werden. Insbesondere am kritischen Übergang in die Sekundarstufe I finden vermehrt Wechsel in das Förderschulsystem statt (z. B. Goldan und Kemper 2019; Klemm 2015, 2018; Kroworsch 2019), d.h. trotz schulischer Inklusion werden die vorhandenen Angebotsstrukturen weiter ausgelastet (Orthmann Bless 2007).

\subsection{Diagnostik sonderpädagogischer Förderbedarfe aus schulsystemischer Sicht}

Bei der formalen Feststellung des Bedarfs sonderpädagogischer Förderung handelt es sich um einen administrativen Prozess, an dem von der Einleitung des Verfahrens bis zur Entscheidung über einen vorhandenen Förderbedarf unterschiedliche Akteure (z. B. Schulamt, Lehrkräfte der Regel- und Förderschulen, Eltern) beteiligt sind und mündet in eine Entscheidung, ob ein Förderbedarf vorliegt oder nicht. Das Verfahren unterscheidet sich nicht nur auf Ebene der Bundesländer (für NRW: Ausbildungsordnung sonderpädagogische Förderung AO-SF), sondern ist z. T. auch auf Ebene der Schulaufsichtsbehörden unterschiedlich geregelt (Neumann und Lütje-Klose 2020). Daneben bindet der Begutachtungs- und Entscheidungsprozess ein erhebliches Maß an Zeit und Ressourcen (Iskenius-Emmler et al. 2003).

Aus systemtheoretischer Sicht können die formalen Diagnosen, auch im inklusiven Unterricht, der Stabilisierung des Regelschulsystems und der Reduktion von Komplexität dienen, z.B. indem den entsprechend diagnostizierten Schülerinnen und Schülern zusätzliche (sonderpädagogische) Unterstützung und Ressourcen zukommen. Zudem erlaubt die amtliche Diagnose eines SPF in ausgewählten För- 
derschwerpunkten die Unterrichtung nach einem zieldifferenten Lehrplan, d.h. die Anforderungen des Regelschulsystems werden ausgesetzt, was eine zusätzliche Entlastung bedeuten kann. Als ultima ratio, wenn die Unterstützungsmaßnahmen des Regelschulsystems den Schülerinnen und Schülern nicht mehr gerecht werden, können diese in das Förderschulsystem wechseln, allerdings erst dann, wenn der Förderbedarf amtlich festgestellt wurde (Neumann und Lütje-Klose 2020).

Aus diagnostischer Sicht sind die Feststellungskriterien der amtlichen Kategorien sonderpädagogischer Förderbedarfe, insbesondere für die unter den Lern- und Entwicklungsstörungen (LES) zusammengefassten Förderschwerpunkte Lernen, Emotional-soziale Entwicklung und Sprache ( $\$ 3$ AO-SF NRW), unpräzise, d.h. eine Differenzlinie zwischen ,Förderbedarf‘ vs. ,kein sonderpädagogischer Förderbedarf ‘ ist nicht eindeutig zu ziehen (Blanck 2020; Grünke und Grosche 2014; Katzenbach 2015; Kottmann 2007; Schlee 2004; Topsch 1975; Werning und Lütje-Klose 2016). Symptomatisch dafür sind die seit Jahrzehnten feststellbaren Unterschiede in der Prävalenz von Förderbedarfen auf Bundes-, Länder- und kommunaler Ebene (z. B. Autorengruppe Bildungsberichterstattung 2014, 2020; Cloerkes 2007; Goldan und Kemper 2019; Katzenbach 2015; Kemper und Goldan 2019; Klemm 2014). Die vagen diagnostischen Kriterien sonderpädagogischer Förderbedarfe verschaffen einen Ermessenspielraum bei der Feststellung von Förderbedarfen, wodurch eine „Ausrichtung an Systeminteressen [...] möglich“ wird (Orthmann Bless 2007, S. 96).

Dieser Ermessensspielraum kann insofern für systemgeleitete Interessen genutzt werden, als dass Entlastungs- und Angebotsstrukturen in Abhängigkeit ihrer Kapazitäten und ihrer räumlichen Nähe eine erhöhte Nachfrage generieren können, was Orthmann Bless (2007, S. 100) als „Sogeffekt“ bezeichnete. Verschiedene Studien konnten den Zusammenhang zwischen dem Vorhandensein von Förderschulstrukturen und ihrer Inanspruchnahme belegen und zwar insbesondere dort, wo diese „schulnah oder schulintern zur Verfügung stehen“ (Bless 2002, S. 69). Bless (2002) kam zu dieser Einschätzung auf Grundlage der Analyse von Exklusionsquoten, Klassenwiederholungen und der Entwicklung zusätzlicher sonderpädagogischer Ressourcen in der Regelschule. Auch Weishaupt (2018) stellt in einer deskriptiven Analyse amtlicher Schulstatistiken die Vermutung auf, dass Unterschiede in den Förderschulbesuchsquoten auf „unterschiedliche Angebotsstrukturen“ (S. 344) zurückzuführen sein könnten. Mit Blick auf Förderschulen bedeutet dies, ,dass dort, wo es mehr Sonderschulen [...] gibt, auch mehr Schüler gesucht und gefunden werden, die diese Schule besuchen müssen." (Werning und Lütje-Klose 2016, S. 72)

$\mathrm{Ob}$ die Nähe zu einer Förderschule einen Einfluss auf die Zahl der diagnostizierten Schülerinnen und Schüler hat, ist bisher nur deskriptiv erforscht worden. Topsch (1975) zeigte für verschiedene Schulverwaltungsbezirke des Bundeslands Nordrhein-Westfalen, dass die Prävalenz von Schülerinnen und Schülern mit diagnostiziertem Förderbedarf im Bereich Lernen enorm variierte (zwischen 1,8 und $16,5 \%)$ und die Zahl der entsprechenden Schülerinnen und Schüler dort besonders hoch war, wo sich auch Förderschulen mit einem entsprechend hohen Platzangebot befanden. In einer Analyse schulstatistischer Daten des Bundesgebiets mutmaßte Cloerkes (2007), dass die enorm variierende Zahl der Schülerinnen und Schüler an Förderschulen vom Platzangebot an diesen abhängt und vermutet, dass sich die Varianz in den Förderquoten auf Ebene der Bundesländer dadurch erklären lässt. 
„Ein bereits vorhandenes Angebot an Schulen und Lehrern muß ausgelastet werden und schafft sich so eine überhöhte Nachfrage." (Cloerkes 2007, S. 25) Bei den genannten Studien handelt es sich allerdings um deskriptive Analysen und nicht um Modelle, die konfundierende Einflussfaktoren, wie beispielsweise den sozio-ökonomischen Hintergrund oder den Anteil nicht-deutscher Schülerinnen und Schüler kontrollieren.

\subsection{Fragestellung}

In der vorliegenden Studie wird erstmalig empirisch-analytisch anhand der amtlichen Schulstatistik für das Land Nordrhein-Westfalen überprüft, ob die Nähe zu einer Förderschule einen systematischen Einfluss auf die Zahl der diagnostizierten Schülerinnen und Schüler an den umliegenden Grundschulen hat. Dabei operationalisieren wir die Nähe zu einer Förderschule, d.h. die vorhandene Angebotsstruktur, als räumliche Entfernung, d. h. wie viele Kilometer eine Grundschule von der nächstgelegenen Förderschule entfernt ist, und prüfen den Effekt dieser Distanz auf den Anteil von Schülerinnen und Schülern mit Förderbedarf an den Grundschulen. Es wird erwartet, dass der Anteil der Schülerinnen und Schüler mit einem formal diagnostizierten Förderbedarf an Grundschulen im Mittel umso höher sein sollte, je geringer die räumliche Distanz zur nächstgelegenen Förderschule ist. Diese Hypothese testen wir sowohl für die Förderbedarfe insgesamt, als auch differenziert für die LES, da deren diagnostische Kriterien als unpräzise kritisiert werden und somit einer Ausrichtung an Systeminteressen besonders Vorschub leisten könnten.

Der Fokus der Studie liegt auf den LES an Grundschulen, weil diese Förderschwerpunkte die deutliche Mehrheit aller Schülerinnen und Schüler mit SPF ausmachen und weit überwiegend im Verlauf der Grundschulzeit festgestellt werden (,grundschulnahe Förderschwerpunkte“; Kottmann 2007, S. 100). Zudem erlaubt die Betrachtung der Grundschulen die Annahme der wohnortnahen Beschulung von Schülerinnen und Schülern (Goldan und Kemper 2019; Makles 2013), sodass sich auf Basis zusätzlicher Variablen die sozio-ökonomische Zusammensetzung der Schülerschaft approximieren und in den Modellen berücksichtigen lässt.

\section{Methode}

\subsection{Daten}

Zur Überprüfung des Effekts werden die Daten der amtlichen Schulstatistik für NRW herangezogen und sekundäranalytisch ausgewertet. Das Flächenland NRW bietet sich für eine Analyse an, da es sowohl Städte als auch weniger dicht besiedelte Regionen vereint und zudem das bevölkerungsreichste deutsche Bundesland ist. Weiter gehört es zu den Bundesländern, welche im Zuge der schulischen Inklusion sowohl inklusive Schulen als auch Förderschulen als Förderorte aufrechterhalten.

Die Analysen beziehen sich auf alle Grundschulen in NRW im Zeitverlauf von 2010/11 bis 2017/18. Für diese insgesamt acht Schuljahre wurden folgende Informationen auf Einzelschulebene berücksichtigt: die Schülerzahlen (differenziert 
nach Zahl der nicht-deutschen Schülerinnen und Schüler und nach amtlich diagnostiziertem Förderschwerpunkt), der Status der Schulen (öffentlich vs. privat) und die Trägerschaft der öffentlichen Schulen (römisch-katholische, evangelische und Gemeinschaftsgrundschulen). Zudem erlauben die Daten eine Differenzierung nach Schulträgern (Gemeinden). Für das Jahr 2017/18 liegen die Postadressen aller Schulen und Schulformen vor, aus denen Distanzmaße berechnet wurden. Entsprechend konnten nur jene Grundschulen in die Analysen aufgenommen werden, die im Jahr 2017/18 noch im Schulbetrieb waren (die Zahl der Grundschulen sinkt über die Schuljahre von 3173 im Schuljahr 2010/11 auf 2787 im Schuljahr 2017/18; d. h. in Summe fließen 22.047 Beobachtungen unter Kontrolle der Schuljahre in die Analysen ein). Da die Anzahl der Förderschulen über die acht Schuljahre hinweg kontinuierlich abnimmt (von 690 im Schuljahr 2010/11 auf 484 im Schuljahr 2017/18) und ein möglicher Effekt dadurch tendenziell eher unter- als überschätzt wird, erscheint es gerechtfertigt, diese Daten unter Kontrolle der Schuljahre dennoch aufeinander zu beziehen.

\subsection{Statistische Analysen}

Zur Berechnung der abhängigen Variablen wurden auf Ebene der einzelnen Grundschulen die Anteile der Schülerinnen und Schüler mit einem Förderbedarf (insgesamt sowie differenziert nach Förderbedarfen) an der Gesamtzahl der Schülerinnen und Schüler berechnet. Auf Basis der Postadressen der Schulen wurden zudem die euklidischen Distanzen, d.h. die Luftliniendistanz in Metern, jeder Grundschule zu jeder Förderschule berechnet (ohne Förderschulen für Kranke). Jeder Grundschule wurde daraufhin die geringste Distanz zur nächsten Förderschule als neue Variable zugewiesen.

Um den Effekt möglichst genau zu schätzen, wurden verschiedene, konfundierende Variablen in die Modelle aufgenommen. Zunächst wurde der Anteil nichtdeutscher Schülerinnen und Schüler (laut amtlicher Statistik) als Kontrollvariable bestimmt. Diese Variable ist von Bedeutung, da der Anteil nicht-deutscher Schülerinnen und Schüler mit der Zahl der Kinder mit einem Förderbedarf vor allem im Bereich Lernen hoch korreliert ist (Kornmann 2013; Kölm et al. 2019). Zusätzlich wird die Trägerschaft der Schulen berücksichtigt, da sich die Zusammensetzung der Schülerschaft in Abhängigkeit von dieser unterscheidet (Makles 2013).

Um zu kontrollieren, ob weitere inklusive Grundschulen in der Umgebung sind, d.h. an denen mindestens ein Kind mit festgestelltem sonderpädagogischen Förderbedarf registriert ist, wurde für jede Grundschule berechnet, wie viele inklusive Grundschulen sich in einem Radius von 2000 Metern befinden. Die Variable dient der Kontrolle eines Selektionseffekts. Ein solcher könnte dann vorliegen, wenn keine weiteren Schulen in der Umgebung inklusiv unterrichten und ggf. alle diagnostizierten Schülerinnen und Schüler auf diese spezielle inklusive Schule gehen bzw. dorthin wechseln müssen. Sie würden dann nicht zufällig, d.h. entsprechend der Wohnortnähe diese Schule besuchen und die Ergebnisse wären verzerrt, wenn diese Schulen sich überzufällig häufig in der Nähe von Förderschulen befinden.

Zur weiteren Kontrolle möglicher konfundierender Variablen wurden für die Studie sozioökonomische Daten auf Rasterebene des RWI-Leibniz-Instituts für Wirt- 
schaftsforschung (Breidenbach und Eilers 2018) einbezogen. Bei den Rastern (Grids) handelt es sich um zeitkonstante Raumeinheiten in Form quadratischer Gitternetze. Sie umfassen eine Fläche von $1 \mathrm{~km}^{2}$ und sind losgelöst von administrativen Grenzen (Budde und Eilers 2014). Elf Variablen wurden anhand einer Hauptkomponentenanalyse mit Varimax-Rotation faktorisiert (Bartlett $\chi^{2}(55)=244.000, p<0,001$; $\mathrm{KMO}=0,86$ ). Nach dem Kaiser-Guttman-Kriterium konnten zwei Faktoren identifiziert werden (Faktor I: Eigenwert $=3,99$, erklärte Varianz $=36,3 \%$, Faktor II: Eigenwert $=3,53$, erklärte Varianz $=32,1 \%$ ). Faktor I umfasst dabei fünf sozio-ökonomische Variablen (Kaufkraft, Arbeitslosenquote, hohes und niedriges Zahlungsausfallrisiko, Ausländeranteil) und Faktor II Variablen, welche die Besiedelungsdichte abbilden (Einwohnerzahl, Zahl der Haushalte, Anteil Kinder pro Haushalt, PKW-Dichte, Haustypen: Anteil Einfamilienhäuser vs. Anteil Häuser mit mehr als 19 Haushalten). Anschließend wurden die Faktorwerte der beiden Hauptkomponenten berechnet und als unabhängige Variablen in das Modell aufgenommen. Da die Daten nur bis zum Jahr 2016 vorliegen, wurden die Werte für das Jahr 2017 auf Basis des Vorjahrs fortgeschrieben.

Um Artefakte der Analysen ausschließen zu können, wurden ebenso die Distanzen zum nächstgelegenen nicht-inklusiven Gymnasium errechnet, dessen Distanz theoretisch keinen Effekt auf die abhängige Variable erwarten lässt (Robustheitsmaß).

Zur Hypothesentestung werden Tobit-Regressionsmodelle unter Berücksichtigung der Mehrebenenstruktur der Daten geschätzt. Ein linkszensiertes Tobit-Regressionsmodell (Amemiya 1985) ist indiziert, da die abhängige Variable - der Anteil der Schülerinnen und Schüler mit Förderbedarf an der Grundschule - insofern beschränkt (,truncated variable“) ist, als dass einige Grundschulen hier in einzelnen Jahren den Wert null aufweisen. Da die betrachteten Schulen in Gemeinden genestet sind, die sich hinsichtlich verschiedener sozio-demografischer Variablen voneinander unterscheiden, sollte für diese nicht beobachtbare Heterogenität kontrolliert werden. Zur Kontrolle der hierarchischen Datenstruktur werden daher Dummy-Variablen für die Gemeinden und die Jahre in das Modell aufgenommen. Die Standardfehler werden zudem auf Ebene der Einzelschulen geclustert, sodass diese als robust gelten können.

\section{Ergebnisse}

\subsection{Deskriptive Ergebnisse}

In Tab. 1 sind zunächst die deskriptiven Statistiken der abhängigen und unabhängigen Variablen dargestellt. Signifikante Mittelwertunterschiede zwischen inklusiven und nicht-inklusiven Grundschulen sind fett gedruckt dargestellt.

Das Minimum aller abhängigen Variablen beträgt 0 (,truncated variable“) und das Maximum bis zu 29,5\%, d.h. dass es einzelne Grundschulen gibt, wo ein erheblicher Anteil der Schülerschaft einen Förderbedarf aufweist. Aufgrund der in einigen Fällen sehr hohen Anteile wurde auf Basis der Daten, welche im Rohformat auch auf Klassenebene vorliegen, geprüft, ob es sich dabei um Schulen handelt, die sepa- 
Tab. 1 Deskriptive Statistik der Grundschulen (arithmetische Mittel) der abhängigen (AV) und unabhängigen Variablen (UV), insgesamt und differenziert nach Schulen mit und ohne Gemeinsamen Lernens (GL)

\begin{tabular}{|c|c|c|c|c|}
\hline & \multicolumn{4}{|c|}{ Arithmetisches Mittel (SD) } \\
\hline & [Min; Max,] & Insgesamt & $\begin{array}{l}\text { Grund- } \\
\text { schulen } \\
\text { ohne GL }\end{array}$ & $\begin{array}{l}\text { Grundschulen } \\
\text { mit GL }\end{array}$ \\
\hline \multicolumn{5}{|l|}{ AVs } \\
\hline $\begin{array}{l}\text { Anteil der SuS mit FSP insgesamt (in } \\
\%)\end{array}$ & {$[0 ; 29,5]$} & $2,46(3,4)$ & - & - \\
\hline $\begin{array}{l}\text { Anteil der SuS mit FSP im Bereich } \\
\text { der LES (in \%) }\end{array}$ & {$[0 ; 27,3]$} & $1,99(2,8)$ & - & - \\
\hline $\begin{array}{l}\text { Anzahl der SuS mit FSP Lernen (in } \\
\%)\end{array}$ & {$[0 ; 14,8]$} & $0,84(1,4)$ & - & - \\
\hline $\begin{array}{l}\text { Anzahl der SuS mit FSP Emotional- } \\
\text { soziale Entwicklung (in \%) }\end{array}$ & {$[0 ; 13,7]$} & $0,61(1,2)$ & - & - \\
\hline $\begin{array}{l}\text { Anzahl der SuS mit FSP Sprache (in } \\
\%)\end{array}$ & {$[0 ; 16,9]$} & $0,54(1,0)$ & - & - \\
\hline \multicolumn{5}{|l|}{ UVs } \\
\hline $\begin{array}{l}\text { Distanz zur nächstgelegenen } \\
\text { Förderschule (in Km) }\end{array}$ & {$[0 ; 29,9]$} & $\begin{array}{l}3,41 \\
(3,1)\end{array}$ & $\begin{array}{l}\mathbf{2 , 9 8} \\
(2,4)\end{array}$ & $\begin{array}{l}\mathbf{3 , 4 9} \\
(3,2)\end{array}$ \\
\hline $\begin{array}{l}\text { Anteil nicht-deutscher Schüler*innen } \\
\text { (in \%) }\end{array}$ & {$[0 ; 95,6]$} & $\begin{array}{l}9,14 \\
(11,1)\end{array}$ & $\begin{array}{l}7,87 \\
(9,6)\end{array}$ & $\begin{array}{l}\mathbf{9 , 4 1} \\
(11,3)\end{array}$ \\
\hline $\begin{array}{l}\text { Anzahl der Grundschulen mit GL im } \\
\text { Radius von } 2 \mathrm{Km}\end{array}$ & {$[0 ; 11]$} & $\begin{array}{l}1,99 \\
(2,1)\end{array}$ & $\begin{array}{l}\mathbf{2 , 1 8} \\
(2,2)\end{array}$ & $\begin{array}{l}\mathbf{1 , 9 5} \\
(2,1)\end{array}$ \\
\hline $\begin{array}{l}\text { Zahlungsausfallrisiko hoch (in \% an } \\
\text { allen Einwohnern/GRID) } \\
\text { (Anker-Item Faktor I - sozioökonomi- } \\
\text { sche Variablen) }\end{array}$ & {$[0 ; 100]$} & $\begin{array}{l}24,39 \\
(19,6)\end{array}$ & $\begin{array}{l}22,25 \\
(18,6)\end{array}$ & $\begin{array}{l}\mathbf{2 4 , 8 3} \\
(20,0)\end{array}$ \\
\hline $\begin{array}{l}\text { Zahl der Haushalte } \\
\text { (Anker-Item Faktor II - Variablen der } \\
\text { Besiedelungsdichte) }\end{array}$ & {$[10 ; 12.204]$} & $\begin{array}{l}1681 \\
(1557,8) \\
N=22.047\end{array}$ & $\begin{array}{l}\mathbf{1 8 9 7 , 2} \\
(1879,2) \\
n=3685\end{array}$ & $\begin{array}{l}\mathbf{1 6 3 8 , 2} \\
(1480,7) \\
n=18.362\end{array}$ \\
\hline
\end{tabular}

Hinweise: Grundschulen mit GL, d.h. mindestens ein/e Schüler/in mit Förderbedarf ist an der Grundschule registriert

FSP Förderschwerpunkt, LES Lern- und Entwicklungsstörungen, Min empirisches Minimum, Max empirisches Maximum; Standardabweichung in runden Klammern; Stichprobengröße kann auf Grund fehlender Werte und Anonymisierung von einzelnen GRIDs variieren; fett gedruckte Werte weisen auf signifikante Unterschiede zwischen den Gruppen der Schulen mit und ohne GL hin $(p<0,05)$; Basis sind t-Tests auf Mittelwertunterschiede

Quelle: Eigene Berechnungen auf Basis der amtlichen Schulstatistik NRW und den RWI GEO-GRIDDaten (Breidenbach und Eilers 2018)

rierte Klassen führen, d.h. in denen nur Schülerinnen und Schüler mit Förderbedarf unterrichtet werden. Dies ist in keiner der entsprechenden Schulen der Fall.

Bei der Distanz zur nächstgelegenen Förderschule ist erkennbar, dass sich inklusive und nicht-inklusive Schulen hinsichtlich der Distanz zur nächsten Förderschule signifikant voneinander unterscheiden. Inklusive Schulen sind im Durchschnitt mehr als $500 \mathrm{~m}$ weiter von der nächsten Förderschule entfernt als nicht-inklusive Schulen. Dieses Ergebnis wird von den Ausreißern in der Distanz beeinflusst, d.h. von 
Abb. 1 Boxplot der unabhängigen Variable „Distanz der Grundschule zur nächstgelegenen Förderschule" in Kilometern (Stand: 2017) für NRW. Mittelwert: 3,4 $(\mathrm{SD}=3,1)$; Median: 2,5. Quelle: Eigene Berechnungen auf Basis der Daten der amtlichen Schulstatistik für NRW (Schuljahr 2017/18)

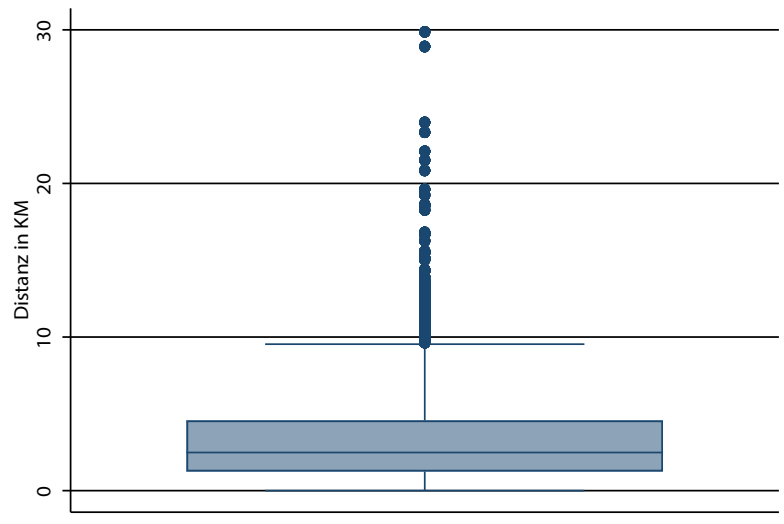

den Schulen, die sich oberhalb des oberen Whiskers (siehe Abb. 1) befinden. Der Unterschied verringert sich unter Ausschluss der Ausreißer deutlich auf $218 \mathrm{~m}$ (Berechnung ohne Abbildung).

Beachtenswert ist ebenso, dass das Minimum der Verteilung bei 0 liegt. Hierbei handelt es sich um insgesamt 17 Grundschulen, welche sich am gleichen Standort befinden wie eine Förderschule, sodass die Distanz den Wert 0 annimmt. Dies wurde anhand der Schulnummern der 17 Schulen im Schulverzeichnis überprüft. Es handelt sich dabei um grundsätzlich unterschiedliche Schulen, deren Anteile an Schülerinnen und Schülern mit Förderbedarfen nicht höher sind als im Mittel.

Mit Blick auf die Faktorvariablen ist in Tab. 1 das sogenannte Anker-Item des jeweiligen Faktors dargestellt, d.h. die Variable, deren Faktorladung den höchsten Wert aufwies und als Stellvertreter für den Faktor betrachtet werden kann.

Die Distanz zur nächsten Förderschule ist in Abb. 1 als Boxplot dargestellt. Dieser lässt auf den ersten Blick erkennen, dass die Distanzen der Grundschulen zur nächsten Förderschule schief verteilt sind, was auf die tendenziell dichte Besiedelung NRWs in Form zahlreicher Großstädte hinweist. Zudem gibt es zahlreiche Schulen, welche die obere Ausreißergrenze (markiert durch den oberen Whisker) überschreiten. Diese Schulen sind zwischen 9,575 Kilometer bis maximal knapp 30 Kilometer von der nächsten Förderschule entfernt. Im Schuljahr 2017/18 betrifft das 151 Schulen. Zur Kontrolle werden alle folgenden Regressionsmodelle daher auch ohne diese Ausreißer geschätzt.

\subsection{Schätzung des Effekts}

Im Folgenden soll für die Grundschulen in NRW der Effekt der Distanz zur nächstgelegenen Förderschule auf den Anteil der Schülerinnen und Schüler mit Förderbedarf an der jeweiligen Grundschule geschätzt werden.

In Tab. 2 ist ein stufenweise aufgebautes Modell für den Anteil der Schülerinnen und Schüler im Bereich der LES abgebildet. Zunächst wurde ein Modell ohne zusätzliche Kontrollvariablen berechnet (wie eingangs beschrieben wurden alle Modelle unter Einschluss der Dummy-Variablen für die Schuljahre und (Schulträger-) Gemeinden berechnet, welche hier nicht separat ausgewiesen werden, da sie ledig- 


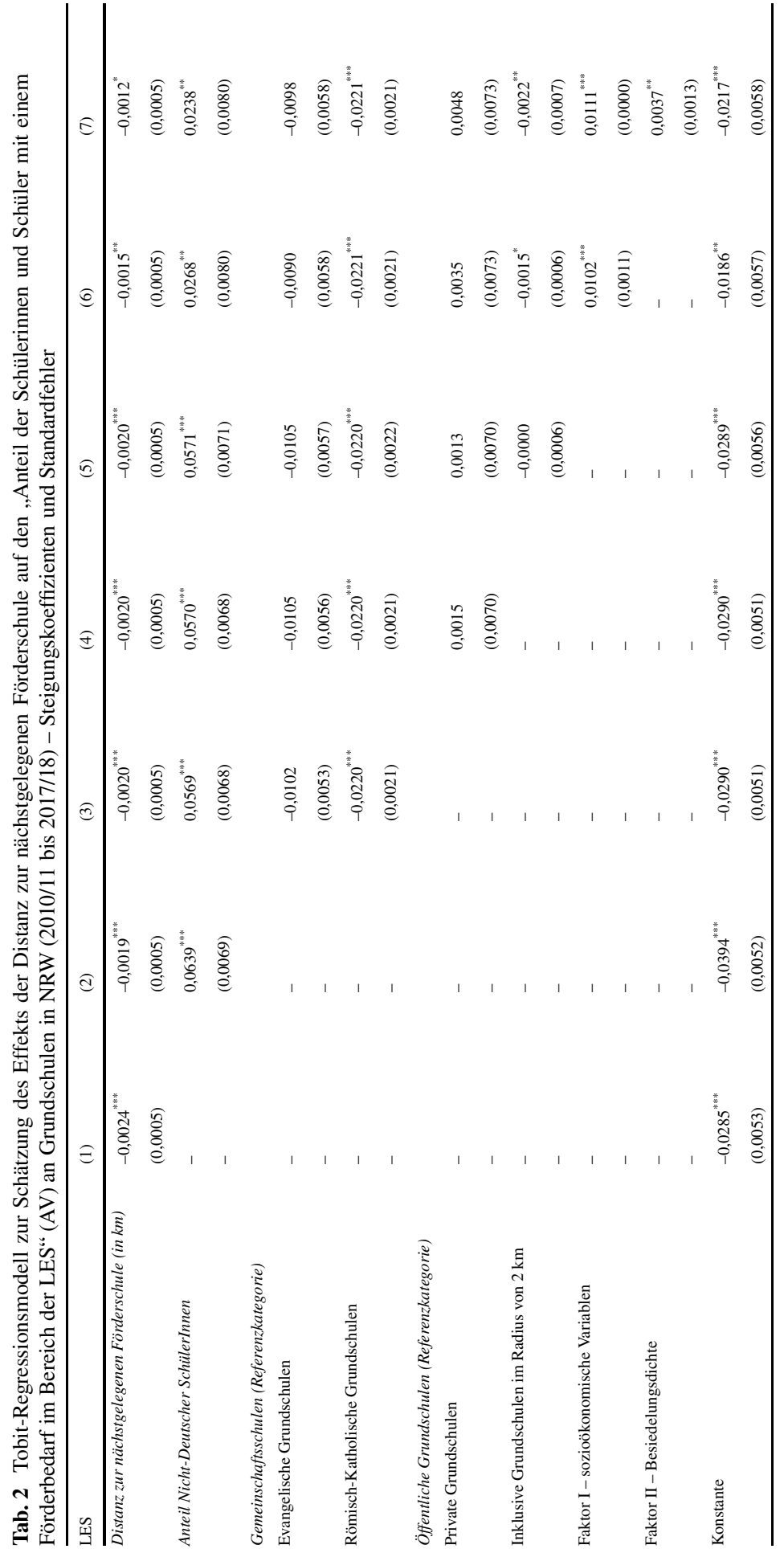




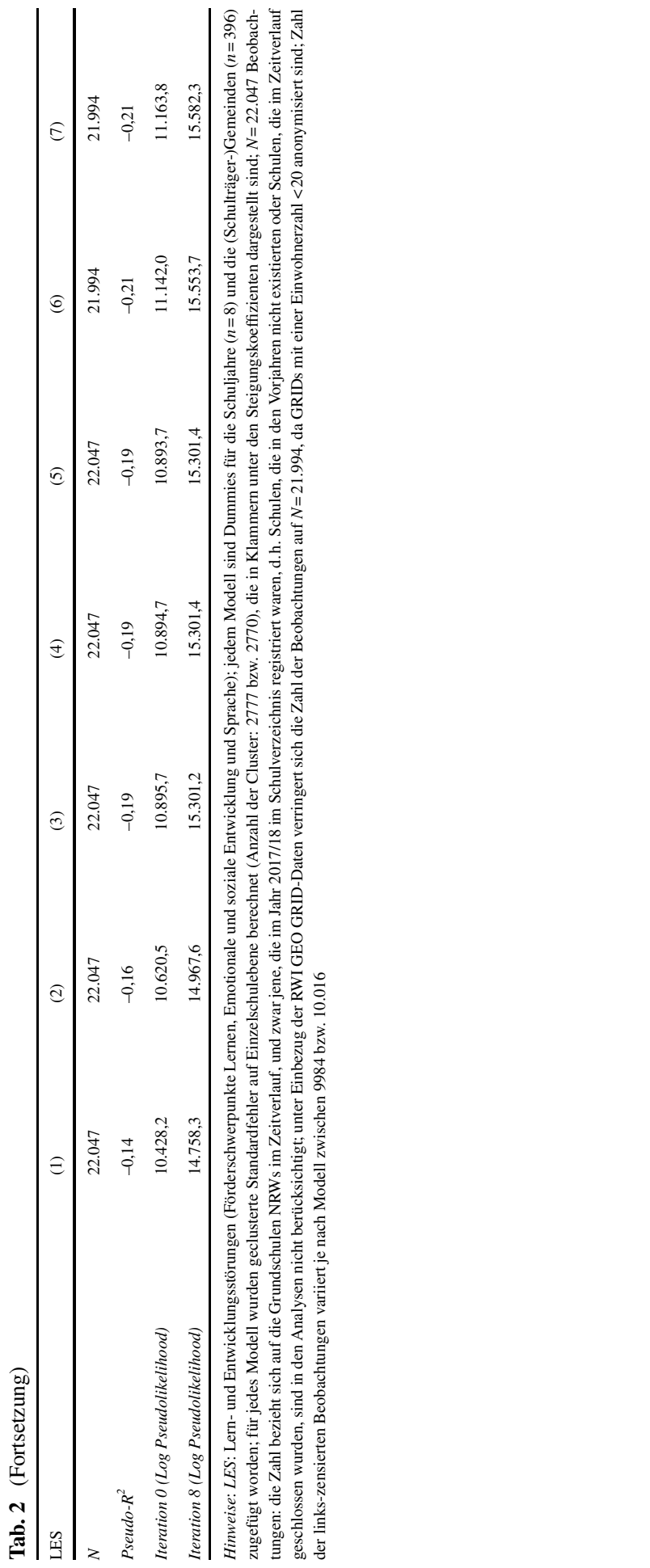




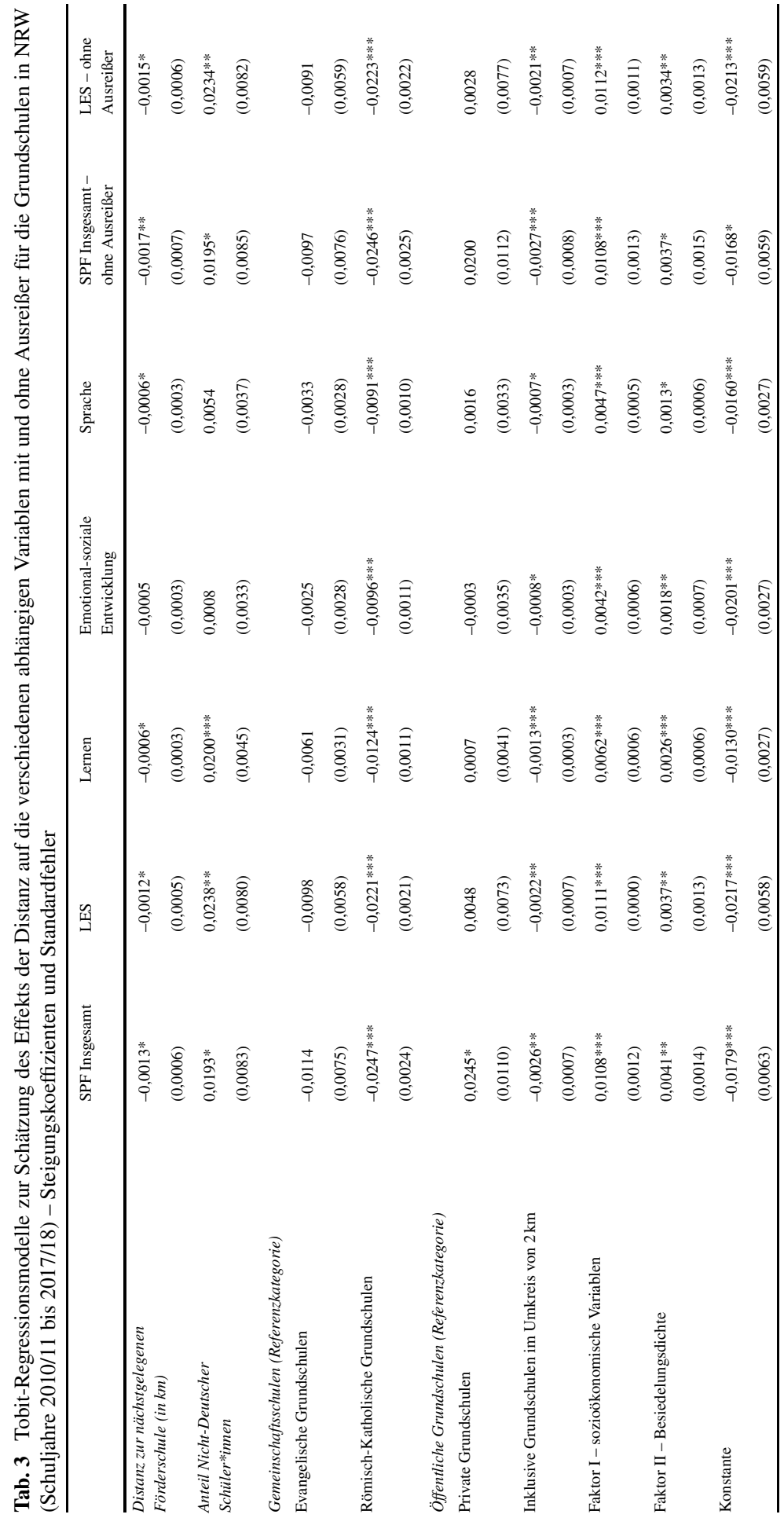




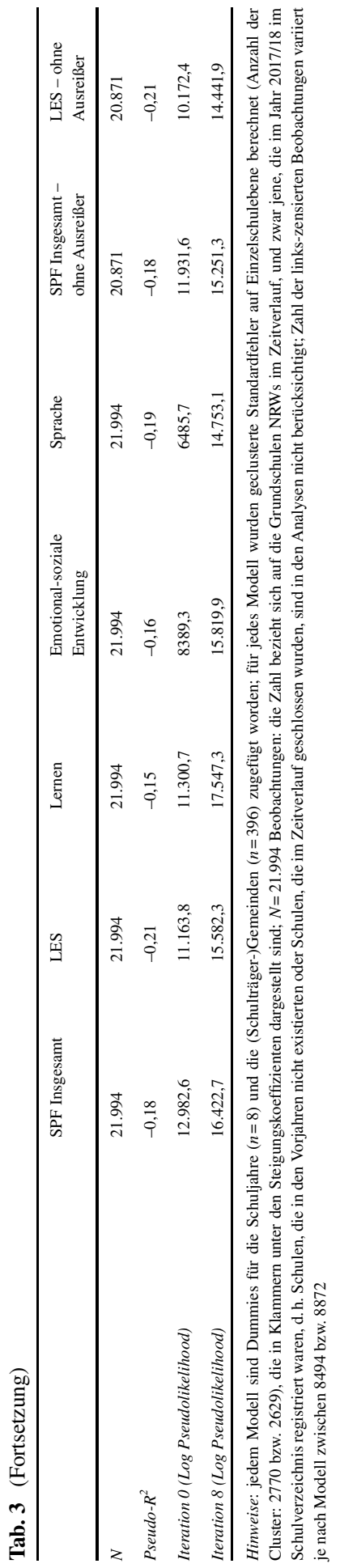


lich der Kontrolle dienen). Es zeigt sich ein signifikant negativer Zusammenhang ( $b=-0,0024 ; p<0,001)$, der folgendermaßen zu interpretieren ist: Wenn die Distanz zur nächstgelegenen Förderschule um einen Kilometer steigt, sinkt der Anteil der Schülerinnen und Schüler mit Förderbedarfen im Bereich der LES an Grundschulen um 0,24 Prozentpunkte.

Im zweiten Modell wird der Anteil nicht-deutscher Schülerinnen und Schüler auf Schulebene einbezogen. Mit Einführung dieser Variable verringert sich der Steigungskoeffizient der Distanzen-Variable deutlich $(b=-0,0019 ; p<0,001)$. Im dritten Modell wird zusätzlich die Trägerschaft der Schule berücksichtigt, wodurch sich der Effekt jedoch nur geringfügig verändert $(b=-0,0020 ; p<0,001)$. Im vierten Modell wird die Schulart und im fünften Schritt die Anzahl der inklusiven Grundschulen im Radius von 2 Kilometern aufgenommen. Beide Kontrollvariablen haben keinen zusätzlichen Einfluss auf die Distanzen-Variable, d.h. der Effekt bleibt unverändert $(b=-0,0020 ; p<0,001)$.

Im sechsten und siebten Modell werden jeweils die Faktorwerte der Variablen für sozioökonomische Kennzahlen (Faktor I) und Variablen der Besiedelungsdichte (Faktor II) aufgenommen. Die Koeffizienten legen nahe, dass beide Variablen mit dem Anteil der Schülerinnen und Schüler mit Förderbedarf im Bereich der LES an Grundschulen konfundiert sind. Der Effekt der Distanz sinkt weiter, bleibt aber signifikant (im sechsten Modell: $b=-0,0015 ; p<0,01$ ). Im Vollmodell (Modell 7) zeigt sich ein signifikant negativer Zusammenhang der Distanz zur nächsten Förderschule auf den Anteil der Schülerinnen und Schüler mit diagnostiziertem Förderbedarf im Bereich der LES an der jeweiligen Grundschule $(b=-0,0012 ; p<0,01)$. Wenn die Distanz zur nächsten Förderschule um einen Kilometer steigt, sinkt der Anteil der Schülerinnen und Schüler mit Förderbedarf um 0,12 Prozentpunkte. Setzt man diesen - prima facie schwach wirkenden - Effekt in Bezug zu den Ergebnissen der deskriptiven Statistik, wird deutlich, dass dieser nicht marginal ist: Über alle Schuljahre hinweg weisen die betrachteten Grundschulen einen durchschnittlichen Anteil der Schülerinnen und Schüler mit Förderbedarf im Bereich der LES von 1,99\% auf, d.h. durch einen Anstieg der Distanz zur nächsten Förderschule um nur einen Kilometer, sinkt der Anteil der Schülerinnen und Schüler mit Förderbedarf durchschnittlich um 0,12 Prozentpunkte und somit um 6\%. Steigt die Distanz beispielsweise um $5 \mathrm{~km}$, würde der Anteil der Schülerinnen und Schüler mit Förderbedarf entsprechend um durchschnittliche 0,6 Prozentpunkte bzw. um 30\% sinken.

Das Modell wurde nun auch für die weiteren abhängigen Variablen berechnet. Die abhängige Variable „SPF insgesamt“ umfasst die sonderpädagogischen Förderbedarfe aller Schwerpunkte, d.h. neben den LES noch die Förderschwerpunkte Geistige Entwicklung, Körperlich-motorische Entwicklung, Hören/Kommunikation und Sehen. Die Ergebnisse der insgesamt sieben Vollmodelle sind in Tab. 3 dargestellt. Es zeigen sich - bis auf den Förderschwerpunkt Emotional-soziale Entwicklung $(p=0,056)$ - auch hier signifikant negative Effekte. Der Anteil von Schülerinnen und Schülern mit einem Förderbedarf (bezogen auf alle Förderbedarfe) steigt durchschnittlich um 0,13 Prozentpunkte, wenn sich die Distanz zur nächsten Förderschule um einen Kilometer verringert $(b=-0,0013 ; p<0,05)$. Für den Förderschwerpunkt Lernen $(b=-0,0006 ; p<0,05)$ zeigt sich, dass der Anteil im Mittel um 0,06 Prozentpunkte steigt, wenn die Distanz zur nächsten Förderschule um einen Kilometer 
abnimmt. Das entspricht mit Blick auf den Mittelwert von 0,84\% (vgl. Tab. 1) einem Anstieg von mehr als $7 \%$ pro Kilometer. Für den Bereich Sprache ist der stärkste prozentuale Effekt zu verzeichnen $(b=-0,0006 ; p<0,05)$, der gemessen am Mittelwert einem Anstieg von mehr als $11 \%$ entspricht, wenn die Distanz um einen Kilometer abnimmt. Für die Modelle ohne Ausreißer zeigt sich sogar ein etwas stärkerer Effekt, d.h. der in den bisherigen Analysen nachgewiesene Effekt wird nicht durch die Ausreißer in der Distanz getrieben.

Obgleich der Effekt entsprechend der eingangs formulierten Hypothese in allen möglichen Modell-Variationen stabil erscheint, wurde zur Kontrolle ein RobustheitsCheck durchgeführt. Hierzu wurde die Distanz zur Förderschule durch die Distanz zum nächsten nicht-inklusiven Gymnasium ersetzt. Für dieses Vollmodell zeigte sich wie erwartet kein Effekt.

\section{Diskussion}

\subsection{Zusammenfassung und Einordnung der Befunde}

In der vorliegenden Studie wurde anhand schulstatistischer Daten für das Bundesland NRW erstmalig überprüft, ob die räumliche Nähe zu einer Förderschule einen Einfluss auf die Zahl der Schülerinnen und Schüler mit festgestellten sonderpädagogischen Förderbedarfen an naheliegenden Grundschulen hat. Die Hypothese, dass der Anteil der Schülerinnen und Schüler mit einem formal diagnostizierten Förderbedarf an Grundschulen höher ist, je geringer die Distanz zur nächsten Förderschule ausfällt, konnte in der vorliegenden Studie bestätigt werden: Erhöht sich die räumliche Distanz zur nächsten Förderschule um einen Kilometer, sinkt der Anteil der Schülerinnen und Schüler mit einem Förderbedarf, z. B. im Bereich der LES um durchschnittlich 6\%. Mit steigender Distanz steigt auch der mittlere Effekt - bei 5 Kilometern im Mittel auf 30\% - und kann somit als bedeutsam bezeichnet werden. Dieser Distanz-Effekt wurde auf den Anteil der Schülerinnen und Schüler mit Förderbedarf insgesamt und auch differenziert nach den Förderschwerpunkten Lernen und Sprache gefunden. Der Effekt für den Förderschwerpunkt Sprache ist mit Abstand am stärksten: Hier sinkt im Mittel der Anteil von Schülerinnen und Schülern mit Förderbedarf im Bereich Sprache um $11 \%$, wenn die Entfernung zur nächsten Förderschule um nur einen Kilometer zunimmt, was bei 5 Kilometern mehr Distanz einem durchschnittlichen Absinken des Anteils um 55\% entspricht. Einzig für den Förderschwerpunkt Emotional-soziale Entwicklung lag die Fehlerwahrscheinlichkeit des Effekts knapp über der 5\%-Signifikanz-Hürde. Die Ergebnisse zeigen, dass eine vorhandene Angebotsstruktur in Form von Förderschulen einen systematischen Einfluss auf die Zahl der formal diagnostizierten Schülerinnen und Schüler an den umliegenden Grundschulen hat.

Aus systemtheoretischer Perspektive erscheinen insbesondere zwei Erklärungen schlüssig. Zum einen ist mit der Einführung der schulischen Inklusion ein inklusives Subsystem an den Regelschulen entstanden (Lambrecht 2020). Entsprechend der Systemtheorie (Luhmann 2002) erzeugt ein solches System sich selbst und strebt nach Selbsterhalt, indem Schülerinnen und Schüler als sonderpädagogisch förderbe- 
dürftig ausgewiesen werden und an der Regelschule verbleiben. Schulstatistisch zeigt sich dies an den Inklusionsanteilen, die seit Einführung der schulischen Inklusion sowohl in NRW als auch in den meisten anderen Bundesländern ansteigen (Autorengruppe Bildungsberichterstattung 2020; Goldan und Kemper 2019; Klemm 2015, 2018; Kroworsch 2019). Ein Distanz-Effekt wäre beispielsweise dann zu erwarten, wenn sich an inklusiven Grundschulen vermehrt Fachpersonal der schulischen Inklusion befindet (z.B., weil die Abordnung sonderpädagogischer Lehrkräfte an naheliegende Grundschulen wahrscheinlicher ist), die den Fortbestand des Subsystems schulischer Inklusion sichern. Zudem könnte es sein, dass zwischen inklusiven Grundschulen und naheliegenden Förderschulen ein stärkerer Austausch stattfindet und möglicherweise Kooperationsstrukturen etabliert wurden, sodass eine amtliche Diagnose z. B. den Rückgriff auf dort vorhandene Ressourcen ermöglicht, ohne dass ein Wechsel an die Förderschule stattfindet.

Eine zweite naheliegende Erklärung besteht in der Selektionsfunktion der Diagnosen, die aufgrund des Fortbestands der Förderschulen auch in Zeiten schulischer Inklusion dazu dienen könnten, einen Systemwechsel anzubahnen. Schulstatistisch zeigt sich dies an den Förderschulbesuchsquoten, die seit Einführung der schulischen Inklusion sowohl in NRW als auch in einigen anderen Bundesländern kaum gesunken sind (Autorengruppe Bildungsberichterstattung 2020; Goldan und Kemper 2019; Klemm 2015, 2018; Kroworsch 2019). Förderschulen werden weiterhin in Anspruch genommen und zwar verstärkt dort, wo sie vorhanden sind. Die Diagnosen können dabei zunächst präventiver Natur sein, indem sie den entsprechenden Schülerinnen und Schülern jederzeit im Verlauf ihrer Bildungsbiografie einen Wechsel an eine Förderschule ermöglichen, z.B. wenn das Schulamt, einzelne Lehrkräfte, Schulleitungen, und/oder die Eltern gemeinsam entscheiden, dass die Förderschule den besseren Förderort für das Kind darstellt. Wenn bereits eine Diagnose gestellt wurde, ist ein Übergang an eine Förderschule kurzfristig möglich, während ansonsten von der Einleitung bis zur Feststellung des Förderbedarfs mehrere Monate vergehen.

Vor dem Hintergrund dieser möglichen Erklärungen ist es interessant, dass sich die Effekte für die einzelnen Förderschwerpunkte zum Teil deutlich unterscheiden. Insbesondere für den Förderschwerpunkt Sprache müsste in vertiefenden Studien untersucht werden, warum der Effekt der Distanz zur nächsten Förderschule auf die Diagnose deutlich höher ist als für die anderen Förderschwerpunkte. Möglicherweise ist die Hemmschwelle, diesen feststellen zu lassen und damit potenziell das Angebot einer naheliegenden Förderschule in Anspruch nehmen zu können - sei es durch Schulformwechsel oder ambulante Hilfen an den inklusiven Grundschulen vergleichsweise gering. Zum einen ist der Förderschwerpunkt Sprache - im Vergleich zu den Förderschwerpunkten Lernen und Emotional-soziale Entwicklung gesellschaftlich mit weniger negativen Vorurteilen behaftet und kann im Verlauf der Sekundarstufe I häufig aberkannt werden, wenn der Förderbedarf nicht mehr besteht. Zum anderen lernen die Schülerinnen und Schüler zielgleich nach dem Lehrplan der allgemeinen Schulen. „Eine kurzfristige Separation in der Sprachheilschule stehe diesem Ziel nicht im Wege, denn ihre Konzeption als Durchgangsschule ermögliche eine anschließende Teilhabe am allgemeinen Bildungssystem und böte die Möglichkeit für alle berufsqualifizierenden Abschlüsse.“" (Odenwald 2020, S. 359) Die 
Tatsache, dass sich für den Förderschwerpunkt Emotionale und soziale Entwicklung kein signifikanter Effekt zeigt, könnte durch die mitunter sehr ausgeprägten und überdauernden Störungsbilder der betroffenen Schülerinnen und Schüler erklärt werden (Hillenbrand 2018). Die formale Feststellung eines sonderpädagogischen Förderbedarfs in diesem Bereich hängt möglicherweise stärker vom Bedarf an zusätzlichen Hilfen und Entlastungsstrukturen ab und weniger von der Frage, ob diese in räumlicher Nähe vorhanden sind.

\subsection{Implikationen für die (sonder-)pädagogische Praxis}

Die vorliegende Studie stellt einen weiteren Beleg dafür dar, dass die Wahrscheinlichkeit einen sonderpädagogischen Förderbedarf diagnostiziert zu bekommen, regional ungleich verteilt ist und dies mitunter davon abhängig ist, ob sich eine Förderschule in der Nähe befindet. Dies wirft Fragen der Bildungsgerechtigkeit auf, die hier nur angerissen werden können.

Einerseits kann man argumentieren, dass Schülerinnen und Schüler bedingt durch die räumliche Nähe zu einer Förderschule einen besseren Zugang zu sonderpädagogischer Förderung erhalten, d.h. gegebenenfalls kommt ihnen aufgrund der formalen Diagnose zusätzliche Unterstützung zu, die Schülerinnen und Schüler in größerer Distanz zu einer Förderschule verwehrt sein kann (aber nicht muss). Andererseits kann argumentiert werden, dass das Etikett ,sonderpädagogischer Förderbedarf“" ein Risiko für die Bildungsbiografie darstellen könnte, z. B. aufgrund von Stigmatisierung. Diese gegenläufigen Betrachtungsweisen sind im Kontext der Debatten um Stigmatisierung, Etikettierungs-Ressourcen-Dilemmata und förderorientierter Handlungsplanung altbekannt (Neumann und Lütje-Klose 2020). An dieser Stelle wären Ideen und Maßnahmen zu entwickeln, welche entweder die Feststellungsverfahren durch standardisierte Kriterien objektiver gestalten oder zeigen wie schulische Inklusion so umgesetzt werden kann, dass formale Feststellungsverfahren - bei ausreichender Versorgung mit Ressourcen - obsolet werden.

Auf Ebene der Personen, welche an der Einleitung der Feststellungsverfahren beteiligt sind, regen die Ergebnisse der vorliegenden Studie zur Reflexion an, um im Einzelfall die Motive für eine mögliche Antragstellung zu hinterfragen. Dabei ist abzuwägen, ob der/die Schüler/in von einer Diagnosestellung profitiert oder ob damit verbundene Entwicklungsrisiken überwiegen (Norwich 2014) und welche Rolle systemische Interessen der beteiligten Subsysteme möglicherweise spielen könnten (Orthmann Bless 2007).

\subsection{Stärken und Limitationen der Studie}

In der vorliegenden Studie konnte erstmals umfassend empirisch belegt werden, dass die Zahl formal festgestellter sonderpädagogischer Förderbedarfe in Abhängigkeit von einer vorhandenen Angebots- und Entlastungsstruktur - hier: Förderschulen variiert. Bei der Datenbasis handelt es sich um eine Vollerhebung für das Flächenland NRW, weshalb der Effekt für dieses Bundesland nicht von Stichprobenfehlern beeinflusst ist. Neben den schulstatistischen Informationen wurden sowohl Variablen zur Kontrolle des sozio-ökonomischen Hintergrunds der Bevölkerung und der Besied- 
lungsdichte im Umkreis der Grundschulen genutzt. Insofern kann ausgeschlossen werden, dass der Effekt dadurch zu erklären ist, dass Förderschulen häufiger in sozial randständigen Gebieten oder Ballungszentren gebaut wurden und der erhöhte Anteil der Schülerinnen und Schüler an den naheliegenden Grundschulen dadurch konfundiert ist.

Zudem konnten auf Basis der Daten ebenfalls Modelle berechnet werden, welche die Distanzen zur nächstgelegenen Förderschule differenziert nach Förderschwerpunkt der Schule berücksichtigen, also z. B. der Anteil der Schülerinnen und Schüler mit Förderbedarf im Bereich Sprache zur nächstgelegenen Förderschule im Bereich Sprache. Ein vertiefter Blick in schulstatistische Daten der Förderschulen zeigte jedoch, dass die meisten Förderschulen - unabhängig von ihrer offiziellen Denomination - Schülerinnen und Schüler verschiedenster Förderschwerpunkte unterrichten. Dies kann zum einen auf die Zusammenlegung von Standorten im Verlauf der Schuljahre als auch auf die Problematik zurückzuführen sein, dass multiple Förderbedarfe festgestellt sein können, obgleich in der Schulstatistik nur die primären Förderschwerpunkte erfasst sind. Aufgrund dieser Unschärfe wurden die Förderschulen in den Modellen nicht nach Förderschwerpunkten differenziert, obwohl sich auch signifikant negative Effekte zeigten.

Obgleich die Regressionsmodelle konservativ und unter größtmöglicher Präzision geschätzt wurden, weist die Studie Limitationen auf. Zunächst liegen die Informationen der Distanz zur nächsten Förderschule nur für das Schuljahr 2017/18 vor. Da sich die Zahl der Förderschulen im Zeitverlauf jedoch deutlich verringert hat, ist davon ausgehen, dass der Distanz-Effekt eher unter- als überschätzt wird, weshalb das Vorgehen als vertretbar angesehen wird. Darüber hinaus liegen die sozialräumlichen Daten auf Grid-Ebene nur bis zum Schuljahr 2016/17 vor, d.h. sie mussten für das Schuljahr 2017/18 fortgeschrieben werden, was eine methodische Unschärfe darstellt, die unter Betrachtung der zeitlichen Stabilität der entsprechenden Variablen als vertretbar betrachtet wird. Hier wurden zudem verschiedene Varianten berechnet, u. a. prognostizierte Entwicklungen auf Basis der Vorjahre, welche die Ergebnisse nicht substanziell verändert haben.

Unsere Ergebnisse können nicht ohne weiteres auf andere Bundesländer generalisiert werden, zumal insbesondere die Stadtstaaten andere geografische Eigenschaften aufweisen als die Flächenländer. Darüber hinaus verfolgen die Bundesländer im Bereich schulischer Inklusion unterschiedliche politische Strategien und verfügen über unterschiedlich lange Erfahrung im Bereich integrativer Beschulung. Zukünftig könnte man den Effekt dennoch für andere Bundesländer untersuchen, insbesondere solche, die Schülerindividualdaten erfassen. Dies würde präzisere Analysen auf Individualebene ermöglichen, z. B. hinsichtlich des Wohnorts und des sozioökonomischen Hintergrunds der Schülerinnen und Schüler. Folglich ließe sich differenzierter erforschen, ob und wann die Diagnosen einer Selektion an Förderschulen dienen und welcher Effekt sich zeigt, wenn man die Übergänge von Grundschulen an Förderschulen in Abhängigkeit der Distanz betrachtet. Perspektivisch wäre anhand verschiedener Datensätze auch zu prüfen, ob die öffentlichen Verkehrswege die Distanzen valider abbilden als der euklidische Abstand und den Effekt sogar verstärken könnten. 
Author Contribution JG und MG entwickelten die Forschungsidee und die theoretischen Grundlagen. JG bereitete sämtliche Daten auf und rechnete alle Analysen. JG und MG schrieben gemeinsam das Manuskript, stimmten der finalen Fassung des Manuskripts zu, tragen die gemeinsame Verantwortung für die Publikation und erklären die Absenz von Interessenskonflikten. Eine Drittmittelfinanzierung der Forschungsarbeit bestand nicht. Wir bedanken uns bei Dr. Anna M. Makles für die Erstellung der Variablen zur Berechnung der Distanzen.

Funding Open Access funding enabled and organized by Projekt DEAL.

Open Access Dieser Artikel wird unter der Creative Commons Namensnennung 4.0 International Lizenz veröffentlicht, welche die Nutzung, Vervielfältigung, Bearbeitung, Verbreitung und Wiedergabe in jeglichem Medium und Format erlaubt, sofern Sie den/die ursprünglichen Autor(en) und die Quelle ordnungsgemäß nennen, einen Link zur Creative Commons Lizenz beifügen und angeben, ob Änderungen vorgenommen wurden.

Die in diesem Artikel enthaltenen Bilder und sonstiges Drittmaterial unterliegen ebenfalls der genannten Creative Commons Lizenz, sofern sich aus der Abbildungslegende nichts anderes ergibt. Sofern das betreffende Material nicht unter der genannten Creative Commons Lizenz steht und die betreffende Handlung nicht nach gesetzlichen Vorschriften erlaubt ist, ist für die oben aufgeführten Weiterverwendungen des Materials die Einwilligung des jeweiligen Rechteinhabers einzuholen.

Weitere Details zur Lizenz entnehmen Sie bitte der Lizenzinformation auf http://creativecommons.org/ licenses/by/4.0/deed.de.

\section{Literatur}

Amemiya, T. (1985). Advanced econometrics. Cambridge: Harvard University Press.

Autorengruppe Bildungsberichterstattung (2014). Bildung in Deutschland 2014. Ein indikatorengestützter Bericht mit einer Analyse zur Bildung von Menschen mit Behinderungen. Bielefeld: Bertelsmann.

Autorengruppe Bildungsberichterstattung (2018). Bildung in Deutschland 2018. Ein indikatorengestützter Bericht mit einer Analyse zu Wirkungen und Erträgen von Bildung. Bielefeld: wbv.

Autorengruppe Bildungsberichterstattung (Hrsg.). (2020). Bildung in Deutschland 2020. Ein indikatorengestützter Bericht mit einer Analyse zu Bildung in einer digitalisierten Welt. Bielefeld: wbv.

Blanck, J. M. (2020). Übergänge nach der Schule als „zweite Chance“? Eine quantitative und qualitative Analyse der Ausbildungschancen von Schülerinnen und Schülern aus Förderschulen „Lernen“. Weinheim: Beltz Juventa.

Bleidick, U. (1985). Theorie der Behindertenpädagogik (Handbuch der Sonderpädagogik, Bd. 1). Berlin: Marhold.

Bless, G. (2002). Moderne Leistungsgesellschaft - Krise im öffentlichen Schulwesen? In K. Bundschuh (Hrsg.), Sonder- und Heilpädagogik in der modernen Leistungsgesellschaft (S. 59-72). Bad Heilbrunn: Klinkhardt.

Breidenbach, P., \& Eilers, L. (2018). RWI-GEO-Grid: Socio-economic data on grid level. Jahrbücher für Nationalökonomie und Statistik, 238(6), 609-616. https://doi.org/10.1515/jbnst-2017-0171.

Budde, R., \& Eilers, L. (2014). Soziö̈konomische Daten auf Rasterebene - Datenbeschreibung der microm-Rasterdaten (RWI Materialien 77). Essen: RWI.

Cloerkes, G. (2007). Soziologie der Behinderten. Eine Einführung. Heidelberg: Universitätsverlag Winter.

Goldan, J., \& Kemper, T. (2019). Prävalenz von Schüler/innen mit Förderschwerpunkt Lernen - Regionale und jahrgangsstufenspezifische Disparitäten. Eine exemplarische Analyse für das Land NordrheinWestfalen anhand von Daten der amtlichen Schulstatistik. Sonderpädagogische Förderung heute, 64(3), 302-317.

Grünke, M., \& Grosche, M. (2014). Lernbehinderung. In G. W. Lauth, M. Grünke \& J. C. Brunstein (Hrsg.), Interventionen bei Lernstörungen (S. 76-89). Göttingen: Hogrefe.

Hillenbrand, C. (2018). Förderschwerpunkt Emotional-soziale Entwicklung. In B. Lütje-Klose, T. RieckeBaulecke \& R. Werning (Hrsg.), Inklusion in Schule und Unterricht. Grundlagen der Sonderpädagogik (S. 182-203). Seelze: Klett.

Iskenius-Emmler, H., Nußbeck, S., \& Haustein, S. (2003). Die Verordnung zur Feststellung des Sonderpädagogischen Förderbedarfs (VO-SF): Anspruch und Wirklichkeit. Sonderpädagogik, 33(1), $28-40$. 
Katzenbach, D. (2015). De-Kategorisierung inklusive? Über Risiken und Nebenwirkungen des Verzichts auf Etikettierungen. In C. Huf \& I. Schnell (Hrsg.), Inklusive Bildung in Kita und Grundschule (S. 33-55). Stuttgart: Kohlhammer.

Kemper, T., \& Goldan, J. (2019). Analysen zur Entwicklung der schulischen Inklusion. Potenziale von Daten der amtlichen Schulstatistik am Beispiel von Nordrhein-Westfalen. In D. Fickermann \& H. Weishaupt (Hrsg.), Bildungsforschung mit Daten der amtlichen Statistik (Die Deutsche Schule: Beiheft 14, S. 234-250). Münster: Waxmann.

Klemm, K. (2014). Auf dem Weg zur inklusiven Schule: Versuch einer bildungsstatistischen Zwischenbilanz. Zeitschrift für Erziehungswissenschaft, 17(4), 625-637.

Klemm, K. (2015). Inklusion in Deutschland - Daten und Fakten. Gütersloh: Bertelsmann Stiftung. https://www.bertelsmannstiftung.de/fileadmin/files/BSt/Publikationen/GrauePublikationen/Studie_ IB_Klemm-Studie_Inklusion_2015.pdf. Zugegriffen: 25. Juli 2017.

Klemm, K. (2018). Unterwegs zur inklusiven Schule. Lagebericht 2018 aus bildungsstatistischer Perspektive. Gütersloh: Bertelsmann Stiftung.

Kölm, J., Gresch, C., \& Kuhl, P. (2019). Zuwanderungsbezogene Disparitäten bei der Diagnose eines sonderpädagogischen Förderbedarfs Lernen und der besuchten Schulart. Zeitschrift für Erziehungswissenschaft, 22(4), 771-789. https://doi.org/10.1007/s11618-019-00896-y.

Kornmann, R. (2013). Die Überrepräsentation ausländischer Kinder und Jugendlicher in Sonderschulen mit dem Schwerpunkt Lernen. In G. Auernheimer (Hrsg.), Schieflagen im Bildungssystem (S. 81-95). Wiesbaden: Springer VS.

Koßmann, R. (2019). Schule und „Lernbehinderung“. Wechselseitige Erschließungen. Bad Heilbrunn: Klinkhardt.

Kottmann, B. (2007). Die Feststellung von sonderpädagogischem Förderbedarf: Benachteiligung der Benachteiligten. In I. Demmer-Dieckmann \& A. Textor (Hrsg.), Integrationsforschung und Bildungspolitik im Dialog (S. 99-108). Bad Heilbrunn: Klinkhardt.

Kretschmann, R. (2007). Lernschwierigkeiten, Lernstörungen und Lernbehinderung. In J. Walter \& F. B. Wember (Hrsg.), Sonderpädagogik des Lernens (S. 4-32). Göttingen: Hogrefe.

Kroworsch, S. (2019). Menschen mit Behinderungen in Nordrhein-Westfalen. Zur Umsetzung der UNBehindertenrechtskonvention in den Bereichen Wohnen, Mobilität, Bildung und Arbeit. Berlin: Monitoring-Stelle UN Behindertenrechtskonvention.

Lambrecht, J. (2020). Warum machen wir nicht einfach Inklusion? Entwicklung einer Theorie schulischer Inklusion. Bielefeld: wbv.

Luhmann, N. (2002). Das Erziehungssystem der Gesellschaft. Frankfurt a. M.: Suhrkamp.

Makles, A. (2013). Bildungsinvestitionen und bildungspolitische Maßnahmen im Spannungsfeld von Gerechtigkeit und Effizienz. Wiesbaden: Springer Gabler.

Neumann, P., \& Lütje-Klose, B. (2020). Diagnostik in inklusiven Schulen - zwischen Stigmatisierung, Etikettierungs-Ressourcen-Dilemma und förderorientierter Handlungsplanung. In C. Gresch, P. Kuhl, M. Grosche, C. Sälzer \& P. Stanat (Hrsg.), Schüler*innen mit sonderpädagogischem Förderbedarf in Schulleistungserhebungen. Einblicke und Entwicklungen (S. 3-28). Wiesbaden: Springer VS.

Norwich, B. (2014). Categories of Special educational needs. In L. Florian (Hrsg.), The SAGE handbook of special education (S. 55-71). Los Angeles: SAGE.

Odenwald, S. (2020). Disziplin unter Druck? Eine tentative Diskursanalyse über Inklusion und Professionalisierung im sonderpädagogischen Förderschwerpunkt Sprache. Zeitschrift für Heilpädagogik, 71(7), 355-365.

Orthmann Bless, D. (2007). Das schulsystemische Paradigma. In J. Walter \& F. B. Wember (Hrsg.), Sonderpädagogik des Lernens (S. 93-103). Göttingen: Hogrefe.

Schlee, J. (2004). Lösungsversuche als Problem - Zur Vergeblichkeit der so genannten Förderdiagnostik. In W. Mutzeck \& P. Jogschies (Hrsg.), Neue Entwicklungen in der Förderdiagnostik: Grundlagen und praktische Umsetzungen (S. 23-38). Weinheim: Beltz.

Smeets, E., \& Roeleveld, J. (2016). The identification by teachers of special educational needs in primary school pupils and factors associated with referral to special education. European Journal of Special Needs Education, 31(4), 423-439. https://doi.org/10.1080/08856257.2016.1187879.

Topsch, W. (1975). Grundschulversagen und Lernbehinderung. Essen: Neue Deutsche Schule Verlagsgesellschaft.

Weishaupt, H. (2018). Sonderpädagogische Förderung im Schuljahr 2016/17. Schulverwaltung (Ausgabe Hessen und Rheinland-Pfalz), 23(12), 341-346.

Werning, R., \& Lütje-Klose, B. (2016). Einführung in die Pädagogik bei Lernbeeinträchtigungen. München: Reinhardt. 